\title{
A Modification of the Weeks Method for Numerical Inversion of the Laplace Transform*
}

\author{
By J. N. Lyness and G. Giunta**
}

\begin{abstract}
The Weeks method for calculating numerical values of $f(t)$ from its Laplace transform $F(s)$ involves the numerical evaluation of a set of contour integrals. In this paper, we describe a powerful modification, by means of which results of comparable accuracy can be obtained using approximately half the number of complex function values. This and other possible minor modifications are discussed.
\end{abstract}

1. Introduction. There is a vast literature relating to the numerical inversion of the Laplace transform. Many authors treat this well-known problem in the following context. We can evaluate the numerical value of the Laplace transform function

$$
F(s)=\int_{0}^{\infty} f(t) e^{-s t} d t, \quad \operatorname{Re} s>\sigma_{0},
$$

at any values of $s$ we like. We wish to construct an algorithm which obtains or uses this information to evaluate $f(t)$ at a given selection of values of $t$ (real) to a specified accuracy. One may assume that $f(t)$ is real, in which case $\overline{F(s)}=F(\bar{s})$. In almost all methods, one needs to know (or one has to find out first) the numerical value of the Laplace convergence abscissa $\sigma_{0}$. This may be defined as the limit of the set of values of $\operatorname{Re} s$ for which this integral converges, or alternatively as the maximum of the real parts of the singular points $s_{j}$ of $F(s)$.

A good brief description of the formulas on which the Weeks method [2] is based is given in Piessens and Branders [1]. In order to provide a proper background, we repeat some of these here. Briefly, we expand $f(t)$ in terms of Laguerre functions. Thus, we assume that

$$
f(t)=e^{\sigma t} \sum_{s=0}^{\infty} a_{s} e^{-b t / 2} L_{s}(b t), \quad \begin{array}{ll}
\sigma>\sigma_{0} \\
& b>0 .
\end{array}
$$

It can be shown that such an expansion exists. The major difficulty in exploiting this numerically is to assign values to $b$ and $\sigma$.

Received August 27, 1984; revised September 13, 1985.

1980 Mathematics Subject Classification. Primary 65R10, 44A10.

* This work was supported by the Applied Mathematical Sciences subprogram of the Office of Energy Research. U. S. Department of Energy, under contract W-31-109-Eng-38.

** Present address: Università di Napoli, Dipartimento di Matematica \& Applicazioni, via Mezzocannone 8, 80134 Napoli, Italy. 
Following Piessens and Branders, using elementary analysis one finds from (1.1), (1.2) and standard properties of the Laguerre polynomials, that

$$
\phi(z)=\frac{b}{1-z} F\left(\frac{b}{1-z}-\frac{b}{2}+\sigma\right)
$$

is regular within a circle $|z|<R_{\text {conv }}$ with $R_{\text {conv }} \geqslant 1$, and that the coefficients $a_{s}$ in (1.2) coincide with the Taylor expansion coefficients of $\phi(z)$, viz.,

$$
\phi(z)=\sum_{s=0}^{\infty} a_{s} z^{s}
$$

The Weeks method comprises an algorithm for the numerical evaluation of the coefficients $a_{s}$ in terms of function values of $\phi(z)$. On the basis of $M$ function values of $\phi(z)$, approximations $\tilde{a}_{s}, s=0,1, \ldots, \mu$, are obtained. The corresponding approximation to $f(t)$ given by (1.2) is

$$
\tilde{f}(t)=e^{\sigma t} \sum_{s=0}^{\mu} \tilde{a}_{s} e^{-b t / 2} L_{s}(b t),
$$

and the approximation error is

$$
\frac{\tilde{f}(t)-f(t)}{e^{\sigma t}}=\sum_{s=0}^{\mu}\left(\tilde{a}_{s}-a_{s}\right) e^{-b t / 2} L_{s}(b t)-\sum_{s=\mu+1}^{\infty} a_{s} e^{-b t / 2} L_{s}(b t) .
$$

Naturally, besides the choices for $b$ and $\sigma$, the success or failure of such an algorithm depends on the accuracy of the approximations $\tilde{a}_{s}$, and on how many $(\mu+1)$ of these are calculated. In Section 2, we describe both the standard methods (Weeks [2], Piessens and Branders [1]) and our modification. In subsequent sections we provide estimates for the error (1.6), which reveal that our modification produces results of comparable accuracy with approximately half the number of complex function values.

2. Approximations for $a_{s}$. Both Weeks' method and the present modification may be developed by considering the Cauchy integral representation of the derivative. This is

$$
a_{s}=\frac{\phi^{(s)}(0)}{s !}=\frac{1}{2 \pi i} \int_{C} \frac{\phi(z)}{z^{s+1}} d z, \quad s \geqslant 0,
$$

where $C$ is any contour which includes the origin and does not include any singularity of $\phi(z)$. For various reasons we take $C$ to be a circular contour centered at the origin of radius $r$. Thus,

$$
a_{s}=\frac{1}{2 \pi i} \int_{|z|=r} \frac{\phi(z)}{z^{s+1}} d z=\frac{1}{2 \pi r^{s}} \int_{0}^{2 \pi} \phi\left(r e^{i \theta}\right) e^{-i s \theta} d \theta, \quad s \geqslant 0 .
$$

This integral may be approximated using the $m$-panel trapezoidal rule. For notational convenience we shall take $m$ to be even. We define

$$
a_{s}^{[m, 1]}(r)=\frac{1}{r^{s} m} \sum_{j=1}^{m} \phi\left(r e^{2 \pi i j / m}\right) \exp (-2 \pi i s j / m) .
$$


In our case, $\phi(z)$ is real when $z$ is real and $\phi(\bar{z})=\overline{\phi(z)}$. Thus, the sum in (2.3) requires only $m / 2$ independent evaluations of (both the real and imaginary parts of) $\phi(z)$. We find

$$
\begin{aligned}
a_{s}^{[m, 1]}(r)= & \frac{2}{r^{s} m} \sum_{j=1}^{m / 2} \operatorname{Re}\left(\phi\left(r e^{2 \pi i j / m}\right)\right) \cos (2 \pi s j / m) \\
& +\frac{2}{r^{s} m} \sum_{j=1}^{m / 2} \operatorname{Im}\left(\phi\left(r e^{2 \pi i j / m}\right)\right) \sin (2 \pi s j / m) .
\end{aligned}
$$

Our modification of Weeks' algorithm uses this approximation to $a_{s}$ for $s=$ $0,1, \ldots, m-1$.

The standard approximation used by Weeks and by Piessens and Branders is different from this. We give a brief derivation. Since $\phi(z)$ has no singularities within the circle $|z|=r$, we have, from Cauchy's theorem,

$$
\frac{1}{2 \pi i} \int_{|z|=r} \phi(z) z^{s-1} d z=\frac{r^{s}}{2 \pi} \int_{0}^{2 \pi} \phi\left(r e^{i \theta}\right) e^{i s \theta} d \theta=\begin{array}{ll}
a_{0} & (s=0), \\
0 & (s>0) .
\end{array}
$$

We now combine this result (divided by $r^{2 s}$ ) with (2.2) by taking the sum and the difference. We find

$$
\begin{aligned}
A_{s} & =\frac{1}{2 \pi i} \int_{|z|=r} \frac{\phi(z)}{z}\left(\frac{1}{z^{s}}+\frac{z^{s}}{r^{2 s}}\right) d z=\frac{2}{2 \pi r^{s}} \int_{0}^{2 \pi} \phi\left(r e^{i \theta}\right) \cos s \theta d \theta \\
& =2 a_{s} \quad(s=0), \\
a_{s} & (s>0),
\end{aligned}
$$

and

$$
\begin{aligned}
B_{s} & =\frac{1}{2 \pi} \int_{|z|=r} \frac{\phi(z)}{z}\left(\frac{1}{z^{s}}-\frac{z^{s}}{r^{2 s}}\right) d z=\frac{2}{2 \pi r^{s}} \int_{0}^{2 \pi} \phi\left(r e^{i \theta}\right) \sin s \theta d \theta \\
& =\begin{array}{ll}
0 & (s=0), \\
i a_{s} & (s>0) .
\end{array}
\end{aligned}
$$

Note that because of the symmetry, the final integral in (2.6a) is purely imaginary. When $s>0$, clearly $A_{s},-i B_{s}$ and $a_{s}$ define the same quantity. In all cases,

$$
2 a_{s}=A_{s}-i B_{s} \text {. }
$$

Piessens and Branders set out this minor transformation from $a_{s}$ to $A_{s}$ using slightly different notation. The approximation used by Weeks consists of approximating the integral representation $(2.6)$ for $s=0,1, \ldots, m / 2$ using the $m$-panel midpoint approximation

$$
\begin{aligned}
A_{s}^{[m, 0]}(r) & =\frac{2}{r^{s} m} \sum_{j=1}^{m} \phi\left(r e^{\pi i(2 j-1) / m}\right) \cos (\pi(2 j-1) s / m) \\
& =\frac{4}{r^{s} m} \sum_{j=1}^{m / 2} \operatorname{Re}\left(\phi\left(r e^{\pi i(2 j-1) / m}\right)\right) \cos (\pi(2 j-1) s / m) .
\end{aligned}
$$

The second equality is justified by the fact that $\phi(z)$ is an analytic function, real for real $z$. Thus $\phi(\bar{z})=\overline{\phi(z)}$, so the real and imaginary parts of $\phi\left(r e^{i \theta}\right)$ are symmetric 
and antisymmetric about $\theta=\pi$. Since the cosine factor is symmetric, the second equality follows. We note that this approximation requires $m / 2$ function evaluations of $\operatorname{Re}(\phi(z))$. Since $\phi(z)=b(1-z)^{-1} F\left(b(1-z)^{-1}+\sigma-b / 2\right)$, it requires $m / 2$ function evaluations of both the real and the imaginary parts of $F(s)$. A similar approximation, based on the endpoint trapezoidal rule is

$$
\begin{aligned}
A_{s}^{[m, 1]}(r) & =\frac{2}{r^{s} m} \sum_{j=1}^{m} \phi\left(r e^{2 \pi i j / m}\right) \cos 2 \pi s j / m \\
& =\frac{4}{r^{s} m} \sum_{j=1}^{m / 2} \operatorname{Re}\left(\phi\left(r e^{2 \pi i j / m}\right)\right) \cos 2 \pi s j / m .
\end{aligned}
$$

Precisely similar remarks apply to this approximation. Weeks uses the midpoint approximation (2.8) with $r=1$. Piessens and Branders suggest both, with $r=1$. By reexpressing $\phi(z)$ in the form

$$
\phi\left(e^{i \theta}\right)=\left(\frac{i b}{2} \cot \frac{\theta}{2}+\frac{b}{2}\right) F\left(\frac{i b}{2} \cot \frac{\theta}{2}+\sigma\right),
$$

both authors mask to some extent the simplicity of this approximation.

Specifically, then, the original Weeks method uses approximation (1.5) with $r=1, \mu=m / 2$ and

$$
\begin{aligned}
& \tilde{a}_{0}=\frac{1}{2} A_{0}^{[m, 0]}(r), \\
& \tilde{a}_{s}=A_{s}^{[m, 0]}(r), \quad s=1,2, \ldots, m / 2-1, \\
& \tilde{a}_{m / 2}=\frac{1}{2} A_{m / 2}^{[m, 0]}(r) .
\end{aligned}
$$

Piessens and Branders discuss both this rule and the corresponding endpoint rule; (we refer to this as the adjusted Weeks method; it is precisely as above but with $[m, 1]$ replacing $[m, 0])$.

Our modification uses (1.5) with $r=1, \mu=m-1$ and

$$
\tilde{a}_{s}=a_{s}^{[m, 1]}(r), \quad s=0,1,2, \ldots, m-1 .
$$

Other modifications (not discussed in the literature) would be to use $B_{s}$ in place of $A_{s}$ in (2.10) above, or $a_{s}^{[m, 0]}(r)$ in place of $a_{s}^{[m, 1]}(r)$ in (2.11) above. (In Section 6 we discuss another much less significant modification.) In spite of the fact that $\mu=m / 2$ in (2.10) and $\mu=m-1$ in (2.11), it is the case that all these possibilities require both the real and imaginary parts of $m / 2$ function values of $F(z)$.

3. Expressions for Error Components $\tilde{a}_{s}-a_{s}$. An expression for $a_{s}^{[m, 1]}-a_{s}$ in terms of higher-order coefficients is derived in this section. This turns out to be a finite form of the Poisson Summation Formula ((3.3) below). We also derive the corresponding expression for $A_{s}^{[m, 1]}-a_{s}$ ((3.5) below).

To obtain the Poisson Summation Formula, one may simply substitute the Taylor series

$$
\phi\left(r e^{2 \pi i j / m}\right)=\sum_{k=0}^{\infty} a_{k} r^{k} e^{2 \pi i k j / m}
$$


into the definition

$$
r^{s} a_{s}^{[m, 1]}=\frac{1}{m} \sum_{j=1}^{m} \phi\left(r e^{2 \pi i j / m}\right) \exp (-2 \pi i s j / m), \quad 0 \leqslant s \leqslant m-1 .
$$

The coefficient of $r^{k}$ is clearly

$$
\frac{a_{k}}{m} \sum_{j=1}^{m} e^{2 \pi i(k-s) j / m} .
$$

This sum is zero unless $(k-s) / m$ is an integer, in which case the coefficient is simply $a_{k}$. Thus the Poisson Summation Formula

$$
r^{s} a_{s}^{[m, 1]}=r^{s} a_{s}+r^{s+m} a_{s+m}+r^{s+2 m} a_{s+2 m}+\cdots, \quad 0 \leqslant s \leqslant m-1,
$$

is obtained. This may be considered to be an expression for the discretization error $r^{s}\left(a_{s}^{[m, 1]}-a_{s}\right)$ in a series whose most significant term is $r^{s+m} a_{s+m}$.

We proceed in a similar way to treat

$$
\begin{aligned}
r^{s} A_{s}^{[m, 1]} & =\frac{2}{m} \sum_{j=1}^{m} \phi\left(r e^{2 \pi i j / m}\right) \cos 2 \pi s j / m \\
& =\frac{1}{m} \sum_{j=1}^{m} \phi\left(r e^{2 \pi i j / m}\right)\left(e^{2 \pi i s j / m}+e^{-2 \pi i s j / m}\right) .
\end{aligned}
$$

Substituting the Taylor series (3.1) here, we find that the coefficient of $r^{k}$ is

$$
\frac{a_{k}}{m} \sum_{j=1}^{m}\left(e^{2 \pi i(k-s) j / m}+e^{2 \pi i(k+s) j / m}\right) .
$$

This sum is zero unless either $(k-s) / m$ is an integer or $(k+s) / m$ is an integer. We find, in particular, that

$$
\begin{aligned}
A_{0}^{[m, 1]}= & 2 a_{0}+2 r^{m} a_{m}+\cdots, \\
r^{s} A_{s}^{[m, 1]}= & r^{s} a_{s}+r^{s+m} a_{s+m}+\cdots \\
& +r^{m-s} a_{m-s}+r^{2 m-s} a_{2 m-s}+\cdots, \quad s=1,2, \ldots, m / 2-1, \\
r^{m / 2} A_{m / 2}^{[m, 1]}= & 2 r^{m / 2} a_{m / 2}+2 r^{3 m / 2} a_{3 m / 2}+\cdots .
\end{aligned}
$$

Comparison of (3.3) and (3.5) shows immediately that $a_{s}^{[m, 1]}$ is generally likely to be a much better approximation. If we suppose that $r^{s} a_{s}$ is a decreasing sequence, and consider a value of $s$ between 1 and $m / 2-1$, we see that the most significant term in $r^{s} A_{s}^{[m, 1]}-r^{s} a_{s}$ is $r^{m-s} a_{m-s}$, while the most significant term in $r^{s} a_{s}^{[m, 1]}-r^{s} a_{s}$ is $r^{s+m} a_{s+m}$, which is generally much smaller. Moreover, $r^{s} a_{s}^{[m, 1]}$ is a reasonable approximation to $r^{s} a_{s}$ for $s=0,1, \ldots, m-1$. On the other hand, since $r^{s} A_{s}^{[m, 1]}=$ $r^{m-s} A_{m-s}^{[m, 1]}$, only the first $m / 2$ values of $A_{s}^{[m, 1]}$ are relevant.

The comparison given above provides the underlying reason why one approach is so much better than the other. The theory given below provides a more quantitative comparison.

4. Bounds for $\tilde{f}(t)-f(t)$ when $R_{\text {conv }}>1$. In this section we employ the Poisson Summation Formula to provide error bounds for $\tilde{f}(t)$. Up to this point, our analysis has allowed $R_{\text {conv }}$, the radius of convergence of the Taylor Series of $\phi(z)$, to be arbitrary, though, in fact, it satisfies $R_{\text {conv }} \geqslant 1$. From this point on, we impose the 
restriction $R_{\text {conv }}>1$. Thus, a class of problems (those for which $R_{\text {conv }}=1$ ) is excluded from the subsequent error analysis. This class is, in fact, one for which these methods are quite unsuitable, involving $f(t)$ with singular behavior at $t=0$.

The error expressions are in terms of parameters $K$ and $R$ which arise in the following way.

Theorem 4.1. Cauchy Inequality. For all positive $R<R_{\text {conv }}$, there exists $K$ (depending on $R$ ) such that

$$
\left|a_{s}\right|<K / R^{s} \quad \text { for all } s .
$$

It is a simple matter to place bounds on many relevant quantities in terms of the given $R$ chosen, so that

$$
r<R<R_{\text {conv }}
$$

and the unknown $K$. Thus, using (3.3), we have

$$
\begin{aligned}
\left|a_{s}^{[m, 1]}-a_{s}\right| & =\left|\frac{1}{r^{s}} \sum_{l=1}^{\infty} r^{s+l m} a_{s+l m}\right| \leqslant \frac{K}{r^{s}} \sum_{l=1}^{\infty} \frac{r^{s+l m}}{R^{s+l m}} \\
& =\frac{K}{R^{s}}\left(\frac{r}{R}\right)^{m} \sum_{l=0}^{\infty}\left(\frac{r}{R}\right)^{l m}=\frac{K}{R^{s}}\left(\frac{r}{R}\right)^{m} \frac{1}{1-(r / R)^{m}} .
\end{aligned}
$$

On the other hand, using the three relations in (3.5) successively, we find

$$
\begin{gathered}
\left|\frac{1}{2} A_{0}^{[m, 1]}-a_{0}\right| \leqslant K(r / R)^{m} /\left(1-(r / R)^{m}\right), \\
\left|A_{s}^{[m, 1]}-a_{s}\right| \leqslant \frac{K}{r^{s}}\left(\left(\frac{r}{R}\right)^{s+m}+\left(\frac{r}{R}\right)^{m-s}\right) /\left(1-(r / R)^{m}\right), \\
\quad s=1,2, \ldots, \frac{m}{2}-1, \\
\left|\frac{1}{2} A_{m / 2}^{[m, 1]}-a_{m / 2}\right| \leqslant \frac{K}{r^{m / 2}}\left(\frac{r}{R}\right)^{3 m / 2} /\left(1-(r / R)^{m}\right) .
\end{gathered}
$$

A second application of the same simple technique provides bounds for $(\tilde{f}(t)-f(t)) / e^{\sigma t}$. We use standard bounds on the Laguerre functions, namely

$$
M_{\lambda}(x)=\max _{s \geqslant \lambda}\left|e^{-x / 2} L_{s}(x)\right|
$$

satisfies

$$
M_{\lambda}(x) \leqslant 1 ; \quad M_{0}(x)=1 ; \quad M_{\lambda}(0)=1 .
$$

From (1.6), we have

$$
\left|\frac{\tilde{f}(t)-f(t)}{e^{\sigma t}}\right| \leqslant M_{0}(b t) \sum_{s=0}^{\mu}\left|\tilde{a}_{s}-a_{s}\right|+M_{\mu+1}(b t) \sum_{s=\mu+1}^{\infty}\left|a_{s}\right| .
$$

For our modification, defined in (2.11), we need (4.1) and (4.3). We find

$$
\begin{aligned}
\left|\frac{\tilde{f}(t)-f(t)}{e^{\sigma t}}\right| \leqslant & M_{0}(b t) \sum_{s=0}^{m-1} \frac{K}{R^{s}}\left(\frac{r}{R}\right)^{m} \frac{1}{1-(r / R)^{m}}+M_{m}(b t) \sum_{s=m}^{\infty} \frac{K}{R^{s}} \\
= & M_{0}(b t) K\left(\frac{r}{R}\right)^{m} \frac{1-(1 / R)^{m}}{\left(1-(r / R)^{m}\right)(1-(1 / R))} \\
& +M_{m}(b t) \frac{K}{R^{m}}\left(\frac{1}{1-(1 / R)}\right) .
\end{aligned}
$$


In the subsequent discussion, we shall refer to the pair of terms on the right-hand side of (4.10) as the discretization error bound and the truncation error bound, respectively. We note that the truncation error does not depend on $r$, and contains a $t$-dependent factor $M_{m}(b t)$.

The expressions corresponding to (4.10) for the Weeks method and for the Piessens and Branders variant are more cumbersome. For the variant (defined by (2.10) but with $[m, 1]$ replacing $[m, 0])$ we use (4.1) and (4.4), (4.5), (4.6) to find

$$
\begin{aligned}
& \left|\frac{\tilde{f}(t)-f(t)}{e^{\sigma t}}\right| \\
& \leqslant \frac{M_{0}(b t) K}{1-(r / R)^{m}}\left[\frac{r^{2}}{R^{m / 2+1}} \frac{1-\left(r^{2} / R\right)^{m / 2-1}}{1-r^{2} / R}+\left(\frac{r}{R}\right)^{m} \frac{1-(1 / R)^{m / 2+1}}{1-1 / R}\right] \\
& \quad+M_{m / 2+1}(b t) \frac{K}{R^{m / 2+1}} \frac{1}{1-(1 / R)} .
\end{aligned}
$$

We are primarily interested in these bounds when $r=1$ and $R>1$. In this case both (4.10) and (4.11) simplify significantly. For our modification, we find

$$
\left|\frac{\tilde{f}(t)-f(t)}{e^{\sigma t}}\right| \leqslant \frac{M_{0}(b t) K}{(1-(1 / R)) R^{m}}+\frac{M_{m}(b t) K}{(1-(1 / R)) R^{m}} .
$$

For the adjusted Weeks algorithm, we find

$$
\left|\frac{\tilde{f}(t)-f(t)}{e^{\sigma t}}\right| \leqslant \frac{M_{0}(b t) K}{(1-(1 / R)) R^{m / 2+1}}+\frac{M_{m / 2+1}(b t) K}{(1-(1 / R)) R^{m / 2+1}} .
$$

In interpreting these results, we must remember that we have chosen $R$ arbitrarily between 1 and $R_{\text {conv }}$, but that $K$ depends on $R$ and increases, often indefinitely, as $R$ approaches $R_{\text {conv }}$. The bounds for the modified algorithm for a particular value of $m$ correspond almost precisely to those on the adjusted Weeks algorithm when $m$ is doubled. It appears then from these bounds that the effect of replacing the Weeks algorithm by our modification is to obtain results of the same caliber based on approximately half the number of function values.

5. The Conditioning Error. All the methods mentioned above involve the numerical approximation of the Taylor coefficients $a_{s}$ of the function $\phi(z)$. This is equivalent to numerical differentiation. It is only prudent to verify that such a method is numerically stable.

We shall employ an elementary but realistic model to the true state of affairs. We shall assume that the calculation is based on rounded or truncated approximations $\hat{\phi}_{j}$ to the true function values $\phi_{j}=\phi\left(z_{j}\right)$. These approximations satisfy

$$
\hat{\phi}_{j}=\phi_{j}+\psi_{j} \varepsilon_{N}, \quad\left|\psi_{j}\right| \leqslant 1
$$

where $\varepsilon_{N}$ is the noise level and the values of $\psi_{j}$ are unknown. We shall assume further that the rest of the calculation is carried out exactly. We define $\hat{a}_{s}^{[m, 1]}(r)$ as the same function of $\hat{\phi}_{j}$ as $a_{s}^{[m, 1]}(r)$ is of $\phi_{j}$, and $\hat{f}(t)$ as the resulting approximation to $\tilde{f}(t)$, and calculate expressions for the differences between the calculated and exact quantities. The details of these calculations are elementary. We find, using 
(2.3), that

$$
\hat{a}_{s}^{[m, 1]}(r)-a_{s}^{[m, 1]}(r)=\frac{1}{r^{s} m} \sum_{j=1}^{m}\left(\hat{\phi}_{j}-\phi_{j}\right) e^{-2 \pi i s j / m},
$$

and using (5.1) this gives

$$
\hat{a}_{s}^{[m, 1]}(r)-a_{s}^{[m, 1]}(r)=\tilde{\psi}_{s} \varepsilon_{N} / r^{s},
$$

with $\tilde{\psi}_{s}$ satisfying

$$
\left|\tilde{\psi}_{s}\right|=\frac{1}{m}\left|\sum_{j=1}^{m} \psi_{j} e^{-2 \pi i j s / m}\right| \leqslant \frac{1}{m} \sum_{j=1}^{m}\left|\psi_{j}\right| \leqslant 1 .
$$

Finally, using (1.6) we have

$$
\begin{aligned}
\frac{\hat{f}(t)-\tilde{f}(t)}{e^{\sigma t}} & =\sum_{s=0}^{m-1}\left(\hat{a}_{s}^{[m, 1]}(r)-a_{s}^{[m, 1]}(r)\right) e^{-b t / 2} L_{s}(b t) \\
& =\sum_{s=0}^{m-1} \frac{\tilde{\psi}_{s} \varepsilon_{N}}{r^{s}} e^{-b t / 2} L_{s}(b t) .
\end{aligned}
$$

We shall be interested in values of $r$ equal to or marginally less than 1 . To this end, we set

$$
r=e^{-\delta} \simeq 1-\delta \text {. }
$$

To obtain a "worst possible" effect, we replace $\tilde{\psi}_{s}$ and the Laguerre functions in (5.5) by 1 to obtain

$$
\left|\frac{\hat{f}(t)-\tilde{f}(t)}{e^{\sigma t}}\right| \leqslant \varepsilon_{N} \sum_{s=0}^{m-1} r^{-s}=\varepsilon_{N} \frac{1-r^{m}}{r^{m-1}(1-r)} \simeq m\left(\frac{e^{m \delta}-1}{m \delta}\right) \varepsilon_{N} .
$$

This bound is too pessimistic. A more optimistic impression of the actual state of affairs may be obtained by assuming (incorrectly) that each $\tilde{\psi}_{s}$ is an independent variate, symmetrically distributed, satisfying

$$
E\left(\tilde{\psi}_{s}\right)=0, \quad E\left(\tilde{\psi}_{s}^{2}\right)=\tilde{\sigma}^{2}
$$

Here, $E$ stands for the expected value, and $\tilde{\sigma}$ is the standard deviation (for a uniform distribution $\tilde{\sigma}^{2}=1 / 3$ ). A simple calculation yields

$$
E\left(\left|\frac{\hat{f}(t)-\tilde{f}(t)}{e^{\sigma t}}\right|^{2}\right) \leqslant \sum_{s=0}^{m-1} \frac{\tilde{\boldsymbol{\sigma}}^{2} \varepsilon_{N}^{2}}{r^{2 s}} \simeq \varepsilon_{N}^{2} \tilde{\boldsymbol{\sigma}}^{2} m\left(\frac{e^{2 m \delta}-1}{2 m \delta}\right) .
$$

We now look at (5.3), (5.7) and (5.9). When $r=1$, the conditioning errors are all what one might expect in a normal calculation. The buildup of statistical error is minimal; the standard deviation in the final result exceeds the original noise level by a factor of less than $\sqrt{m}$.

However, when $r<1$, the appearance of terms like $r^{-s}$ in (5.3) and $r^{-(m-1)}$ in (5.7) shows immediately that the noise level may be significantly amplified. When $r=1 / 2,(5.3)$ indicates that three decimal digits are lost when $s=10$, six when $s=20$, and so on, which is often an intolerable situation. However, values of $r$ only marginally less than 1 can be handled, so long as the effect of this source of error is monitored. 
6. Choice of the Parameter $r$. The authors are involved in the construction of numerical software. In a subsequent more technical publication we plan to discuss the choice of the parameters $\sigma, b, m$ and $r$ required to define the method. However, the choice of $r$ is a direct consequence of the foregoing formulas and it seems pertinent to discuss it here.

First, we note that the error bound (4.12) implies that when $r=1$ the truncation and discretization errors are approximately equal. Reducing $r$ has no effect on the truncation error but, in view of (4.10), does reduce the discretization error. Thus, in general, the advantages of using $r<1$ are not great. One reduces only one of two equally large components of the overall error, but introduces a possible numerical instability.

However, one of the function values required using the trapezoidal rule is $\phi(r)$. When $r=1$, reference to (1.3) shows that a limit calculation is required. We have

$$
\operatorname{Lim}_{z \rightarrow 1} \phi(z)=\operatorname{Lim}_{s \rightarrow \infty} s F(s)=f(0)
$$

In general, when $R_{\text {conv }}>1$, there is no actual singularity at $z=1$. It is simply inconvenient to be obliged to provide special programming for this single function value.

One simple way around this difficulty is to use the equally accurate midpoint rule $[m, 0]$ which does not need this function value. In a one-shot calculation, this is the obvious solution. In an automatic algorithm where the value of $m$ is increased by stages until sufficient accuracy has been obtained, this choice introduces another difficulty, related to reuse of function values. Effectively, we need a symmetric rule $[m, 1]$ or $[m, 0]$ to ensure that only $m / 2$ complex function values are required. Using $[m, 1]$, we can proceed by doubling the value of $m$ at each stage. Using [ $m, 0]$, we have to triple the value of $m$ at each stage. The latter choice is ultimately even more wasteful than the earlier choice, as the margin by which we exceed the value of $m$ that is actually required is significantly increased.

To avoid this problem, in our software we have used $r=1-\delta$ with $\delta$ chosen so that the value of $\delta \bar{m} \leqslant 2$ where $\bar{m}$ is the largest value of $m$ allowed by the program.

7. Concluding Remarks. In Sections 1 and 2 we have described the standard Weeks method and our modification. The results of Sections 3 and 4 establish theoretically that our modification, by providing approximations to twice as many expansion coefficients is much more cost effective. Sections 5 and 6 discuss the conditioning error and some of the effects of altering the parameter $r$.

That our modification is in practice as efficient as is indicated by the theory has been confirmed by many numerical experiments. In fact, the importance of the modification was discovered by numerical experiment. Previously, the authors had believed that both methods were equivalent.

In practice, many further decisions are required. These include the choice of $b, \sigma$ and $r$ and the construction of stopping criteria. The present authors are in the process of constructing numerical software for this problem and will present somewhat heuristic recommendations in a future publication devoted to practical matters. The scope of the present paper has been limited to the theoretical aspects of this problem. 
The authors would like to acknowledge the help of Drs. Almerico Murli and L. M. Delves.

Mathematics and Computer Science Division

Argonne National Laboratory

Argonne, Illinois 60439

1. R. Piessens \& M. Branders, "Numerical inversion of the Laplace transform using generalized Laguerre polynomials,” Proc. IEE, v. 118, 1971, pp. 1517-1522.

2. W. T. WEEKS, "Numerical inversion of Laplace transforms using Laguerre functions," J. Assoc. Comput. Mach., v. 13, 1966, pp. 419-429. 